\section{STM Experimenter: Using HyperCard and MacRecorder in short-term memory experiments}

\author{
RICHARD COX and CHARLES HULME \\ University of York, York, England \\ and \\ GORDON D. A. BROWN \\ University of Wales, Bangor, Wales
}

\begin{abstract}
We describe a Macintosh program for the presentation of spoken word lists in short-term memory (STM) experiments. The program was developed with the use of HyperCard and MacRecorder. Parameters such as rate of item presentation, number of items per trial, and number of trials per block may be adjusted by the experimenter. The program features high-quality digitized sound, trial-totrial word list randomization, and data logging. Computer-based experimentation offers several advantages over traditional approaches that involve tape recordings or lists read aloud by the experimenter. Methodological improvements afforded by computer-controlled item administration are discussed.
\end{abstract}

Short-term memory phenomena have been widely studied by experimental psychologists for over a century (Ebbinghaus, 1885; Jacobs, 1887). Computer control has advantages in such experiments, because it ensures that uniform procedures are used across different subjects and experiments and lessens the burden of running the experiments.

The use of computers for running short-term memory experiments is commonplace for visually presented material. Several psychological laboratory packages are available for the Macintosh. MacLaboratory (Chute, 1989 ) is a set of software and teaching resource materials containing demonstrations and experiments in nine areas of psychology. Experiments in Cognitive Psychology (Tversky, 1990) is a set of HyperCard (Version 1.2.2) stacks designed for teaching. The collection of 22 experiments contains nine memory experiments with visually presented verbal stimuli. Other examples of psychology software packages for the Macintosh include PsychLab (Gum \& Bub, 1988) and MacLab (Costin, 1988). In addition, Nason and Zabrucky (1988) and Dougherty (1990) have, respectively, described Macintosh programs for monitoring the comprehension of text and for teaching about subjective contours and other visual illusions.

However, for auditory material, computer-controlled presentation with the Macintosh has not been widespread.

This research was supported by Grant R 000232576 from the Economic \& Social Research Council of the United Kingdom. Address all correspondence to $\mathrm{C}$. Hulme, Department of Psychology, University of York, York YO1 5DD, England.
In such research, word lists are typically presented on a tape recorder. Although tape recordings ensure standard presentation, they can be laborious to use in experiments with a number of conditions with different orders of presentation. Digitized sounds on microcomputers provide much greater flexibility and ease of use. The nontemporal nature of disk storage facilitates the construction of complex stimulus presentation orders and sequences, because, unlike tape-recorded stimuli, the items can be recorded independently of intended presentation order (Gibson, 1987). However, there is little existing Macintosh software for use with auditory stimuli in short-term memory experimentation, in which, for example, a common requirement is that the number of trials per block increase from block to block. MacLab is designed for use with pictorial displays, and although PsychLab can play some types of sound files, it is also mainly designed for use with textual and graphic stimuli or audio tones.

The Macintosh is an eminently suitable microcomputer for use with sound. It is capable of reproducing frequencies up to $11 \mathrm{kHz}$ at 256 sound levels. Recently Reekes and Thompson (1991) have pointed out that Macintosh computers have greater sound resolution than IBM PC or compatible computers ( 8 bit vs. 1 bit) and that, unlike IBM PCs and compatibles, the Macintosh has the requisite software and hardware for easily reproducing complex sounds. Those authors provide a useful general introduction to sound sampling and digitization, as well as to the sound circuitry and software of the Macintosh. They provide a summary of the sound capabilities of various models of Macintosh computers.

The Macintosh is popular for research use because of its graphical user interface and the availability of useful laboratory software. Recently, with the advent of products for sound digitization such as MacRecorder from the Farallon company, the manipulation of digitized sound recordings has become viable. High-quality sound output is now relatively easy to achieve, and high-level object-like programming languages such as HyperCard's HyperTalk have made the development of software easier for psychologists who lack formal training in computer programming. Geisler-Brenstein and Brenstein (1989) provide a recent discussion of the potential of HyperCard for psychological research and instruction. Martin and Allan (1991) used HyperCard and MacRecorder in a comparison of touch-screen, mouse, and voice response inputs by subjects performing a digit-span task. However, Martin and Allan have not provided detailed descriptions of the HyperCard stacks that they developed for the experiments. Gibson (1987) discusses the advantages of using digitized auditory stimuli on the Macintosh. These include simplifying the process of compiling materials and providing a highly flexible digital sound editing environment useful for the production of specialized stimuli such as word fragments. 
Sound editors, such as MacRecorder's SoundEdit program, enable psychologists to prepare types of stimulus materials that previously would have required very expensive digital sound equipment. For example, a sound item's tempo can be manipulated without distorting pitch, nonwords can be constructed from the phonemic elements from real words, and so on. Another possibility is the use of stereo sound on the Macintosh II, IIx, SE/30, and IIcx. Memory experiments involving dichotic listening tasks would be relatively straightforward to prepare.

The HyperCard program described here (STM Experimenter) was developed for the presentation of spoken word and number lists in short-term memory experiments. STM Experimenter is designed to be easy to use, yet flexible. Experimenters can record their own stimuli by using Farallon's MacRecorder or, in the case of newer Macintosh models such as the LC, si, and Classic II, by using the built-in microphone and audio software. Two procedures are commonly used in STM experiments. In a memory span procedure, the experimenter determines the length of a sequence a subject can reliably recall correctly in the order of presentation. In a fixed list length procedure, a list length is used that is usually just above the subject's memory span and the number of items recalled in their correct serial positions over trials is recorded. The program can run experiments using either a memory span or fixed list length procedure.

\section{Hardware}

The hardware used in the development of STM Experimenter consisted of an Apple Macintosh SE/30 with a 40-MB internal hard disk and Farallon Computing's MacRecorder sound digitizer. For higher quality sound reproduction on playback, an external amplifier/speaker unit was plugged into the speaker output jack of the SE/30.

\section{Software Development}

The following software was used in the development of STM Experimenter: HyperCard Version 2.0v2 with the HyperTalk scripting language; Power Tools stack (supplied with HyperCard Version 2.0v2); SoundEdit and Hypersound toolkit (supplied with Farallon Computing's MacRecorder).

STM Experimenter was developed with the HyperTalk programming language of HyperCard 2.0v2. One external function (XFCN) resource was transferred from the Hypersound toolkit supplied with MacRecorder to extend HyperTalk's range of commands and functions. This was the sound list SndList XFCN, which produces a directory of sound resources. When run, the STM Experimenter displays a directory of sounds in the Master List text field (see Figure 1). All fields (Master list, Selection, and Random of selection) permit point-and-click playing of words listed.

STM Experimenter has been tested on several Macintosh models. These include the Classic, LC, SE/30, and IIcx-all running System 6.07, Multifinder, and HyperCard 2.0v2. STM Experimenter ran without problems on all of these maohines. Timing in STM Experimenter is based on the system clock rather than the cycle rate of the CPU, and hence variations from one Macintosh to another were small. The program was also tested on an LC running HyperCard 2.0v2 under System 7.0-again, STM Experimenter ran without problems.

\section{Sound Recording Procedure}

High-quality sound output was considered to be essential, and considerable care was therefore taken to ensure that this was maximized. Spoken word lists were recorded on an Uher 4200 Report Monitor reel-to-reel tape recorder using a Sennheiser MD421N microphone in an acoustically dampened recording chamber. Experience suggets, however, that the acoustically dampened chamber is not essential for recordings of acceptable quality.

The recordings were then played into the MacRecorder digitizer through a direct line-in lead from the Uher's monitor output. Sounds were captured with the MacRecorder SoundEdit software. The input level control on the MacRecorder was adjusted to provide the greatest signal-to-noise ratio possible without distortion. We recommend the use of the $22-\mathrm{kHz}$ digitization rate to maximize clarity. Subjectively, items digitized at $22 \mathrm{kHz}$ are equivalent in fidelity to a good quality tape recording. To date, the authors have collected data from more than 100 subjects ranging from young children to adults and have not experienced any problems with stimulus discriminability.

With the use of SoundEdit software supplied with MacRecorder, the sound samples were saved as sound resources into the STM Experimenter HyperCard stack. With nine one-syllable words transferred, the stack size was $155 \mathrm{kB}(50-\mathrm{kB}$ stack $+105-\mathrm{kB}$ digitized sound resources). The audio output of the Macintosh was amplified and played through an external 8-in. midrange speaker to enhance playback quality and volume.

With some sacrifice of fidelity, the sound files can be compressed to save memory. Alternatively, sounds may be sampled at a lower rate than $22 \mathrm{kHz}$-again with a reduction of sound quality. The MacRecorder SoundEdit and Hypersound software enables sound to be sampled at $22 \mathrm{kHz}$ (highest quality) or downsampled at rates of 11,7 , or $5 \mathrm{kHz}$. Sounds may also be compressed at four ratios. To illustrate the memory savings from downsampling or compression, the words piano, policeman, and banana were spoken at a normal rate (duration = $5.45 \mathrm{sec}$ ), recorded as a single item, and subjected to the various sampling and compression options. The memory storage space for each option was as follows:
22-kHz sampling rate:
$121 \mathrm{~K}$
$11-\mathrm{kHz}$ sampling rate:
$53 \mathrm{~K}$
7-kHz sampling rate:
$5-\mathrm{kHz}$ sampling rate:
3:1 compression ratio:
4:1 compression ratio:
6:1 compression ratio:
$39 \mathrm{~K}$
$28 \mathrm{~K}$
$37 \mathrm{~K}$
$27 \mathrm{~K}$
8.1 compression ratio: $\quad 11 \mathrm{~K}$ 
Subjectively, the $3: 1$ sound compression ratio produces sound of similar quality to sampling at $11 \mathrm{kHz}$. The file size of the former is much less than the latter, however (37K vs. 53K in the case of the test items). For stimulus presentation in short-term memory experiments, a sampling rate of $11 \mathrm{kHz}$ or a 3:1 compression ratio would probably be the lowest acceptable in terms of sound reproduction quality. A demonstration stack that illustrates the same sound sample played at the various sampling rates and compression ratios is available along with STM Experimenter.

The Macintosh LC, si, and Classic II models are supplied with a built-in microphone, sound input jack, and HyperCard audio palette software. The audio palette is supplied in the form of three HyperCard stacks: Appointments with audio, Addresses with audio, and Audio help. When the stacks are used, new items are added to the standard HyperCard Edit menu. The menu items allow the recording, editing, and deletion of sounds and provide a help facility. The audio palette can also be used on other Macintosh models in conjunction with MacRecorder. Version 2.0.3 of the MacRecorder software contains a suitable driver, which must be placed in the system folder. The audio palette software can be used to record sounds for use with STM Experimenter. Two sampling rates (22 kHz "best" and $11 \mathrm{kHz}$ "good") and two compression ratios (3:1 and 6:1) are available. The audio palette can also be used to delete sounds from STM Experimenter. Whenever a sound is added to or deleted from STM Experimenter with the audio palette, the Update list button (Figure 1) can be clicked to amend the Master List field. Small condensor microphones such as those supplied with the LC, si, and Classic II will not, however, provide high fidelity sound recordings, in part because of ambient noise from the computer fan and other sources.

Sound resources can also be moved into or removed from STM Experimenter by using the Resource Mover from the HyperCard 2.0v2 Power Tools utilities. It is also possible to use the Apple Macintosh resource editor (ResEdit) or MacRecorder's SoundEdit. One of the best general utilities currently available seems to be SoundMover (available from Riccardo Ettore, 67 Rue de la limite, $1970 \mathrm{~W}-$ Oppen, Belgium; price, \$25). SoundMover recognizes and converts many different sound file formats, and the documentation contains useful information about using sound with the Macintosh.

\section{Using STM Experimenter}

When STM Experimenter is run, a unique letter is assigned to each of the sound resources in the master list (Figure 1). The randomization routines (activated by dice icon buttons) operate on the letter tags rather than the sound names, to avoid problems if more than one sound resource shares the same name. Other buttons play an entire list (speaker-list icons) or permit user selection of items from the master list (finger-on-page icons). A Rate menu was also added to the Apple Macintosh menu bar. This permits control of the rate at which items are presented within trials (interitem intervals of 1,2 , or $3 \mathrm{sec}$ ). Buttons showing the dice icon randomize (without duplication) either a whole list of items or a randomly selected subsample of user-determined size.

The program can be run in two modes. In the first mode, all parameters (such as list length, the span width, and the rate at which items are presented) can be varied on a trial-by-trial basis by using the on-screen control but-

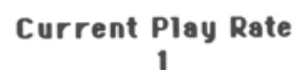

1

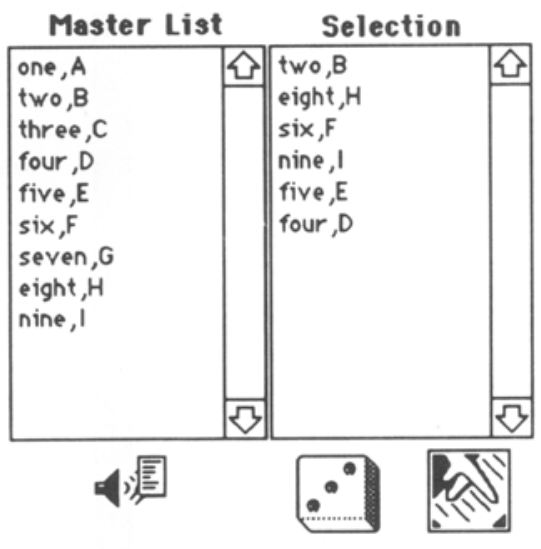

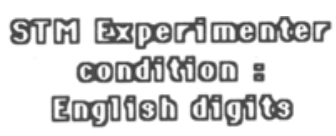

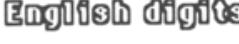
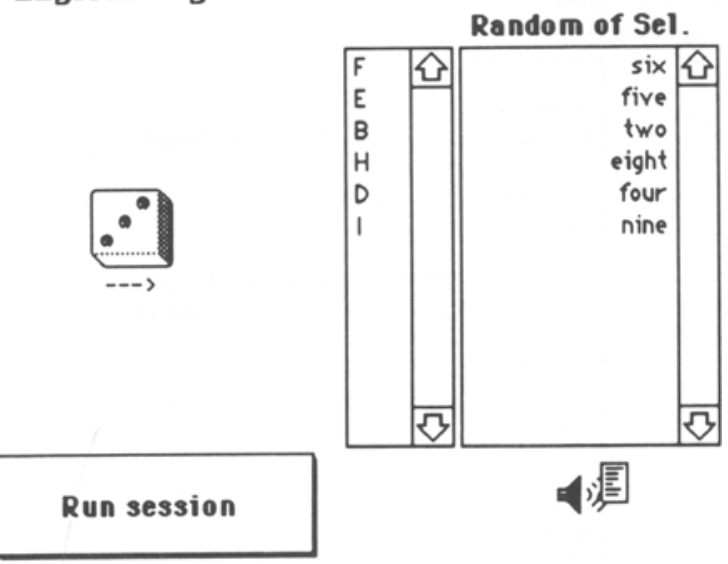
tons described above. The second mode (activated via the Run session button) permits the running of multitrial session blocks and is ideal for experiments involving the measurement of memory span for different sets of materials. The experimenter is prompted for subject, session details, trial details, and item presentation rate. The experimenter selects the number of trials per block (default $=4$ ) and the number of items to be presented in the first block of trials. The program then administers a session in which the number of items per trial is incremented by one on each successive block until the experimenter ends the session. A session can be terminated at the end of any trial block, but it would usually be terminated at the discontinuation point defined as erroneous recall on all the trials of a block at a particular span level. For each trial within and between trial blocks, different random selections of items are selected from the master list.

When one is using STM Experimenter, the computer monitor should be positioned to prevent the subject from seeing the screen, since the stimulus item names are visible (as an aid to the experimenter). On each trial, following item presentation to the subject, the experimenter is presented with a dialog box. The subject's recall performance is indicated by clicking on one of two buttons labeled "correct" and "incorrect." With STM Experimenter, as opposed to traditional methods, the experimenter's cognitive load is reduced because only a single mouse click is required to record the subject's trial performance and a list of the items is visible to the experimenter as a cue. The experimenter's attention is thus freed so that he/she can concentrate on the subject's response. The subject's response, together with a listing of trial items, the date, the time, and the experimental condition are written to a data file at the end of the session. At the conclusion of the session, the span score is computed and written to a file. Figure 2 is an unedited example of the data file output. The data file may be opened, read, and printed from within STM Experimenter. Alternatively, the data file may be opened as a text file by using a word processor. If detailed analyses of subject error patterns are required (i.e., classification as intrusions, omissions, inversions, etc.), testing sessions should be concurrently tape recorded.

To avoid the size of STM Experimenter stacks' becoming too unwieldy, a separate copy of the stack is used for each session condition. For example, the stack shown in Figure 1 contains "English digits." Note that the stack name is shown on screen in the condition text field (Figure 1) and written to the data file under the same heading (Figure 2).

STM Experimenter has several advantages over traditional methods of auditory STM span assessment; these include consistency of item presentation rate, reduced experimenter workload, and therefore, less experimenter error. STM Experimenter also provides an objective computation of span score.

The program can be used for teaching. For example, it could be used to illustrate experimental research methods as well as classic short-term memory phenomena such as the word-length effect (Baddeley, Thomson, \& Buchanan, 1975 ) and the acoustic similarity effect (Conrad, 1963).

\section{Availability}

The authors will supply interested readers with a copy of STM Experimenter and would welcome feedback and comments. The shareware program SoundMover Version 1.7 is also included on the disk as part of the Sound Manager Package (SMP) by Riccardo Ettore (to whom a shareware fee of $\$ 25$ must be sent if SMP is kept for longer than the trial period). A HyperCard demonstration of sound sampling rates and compression ratios developed by the first author is also included. A fee of $£ 5.00$

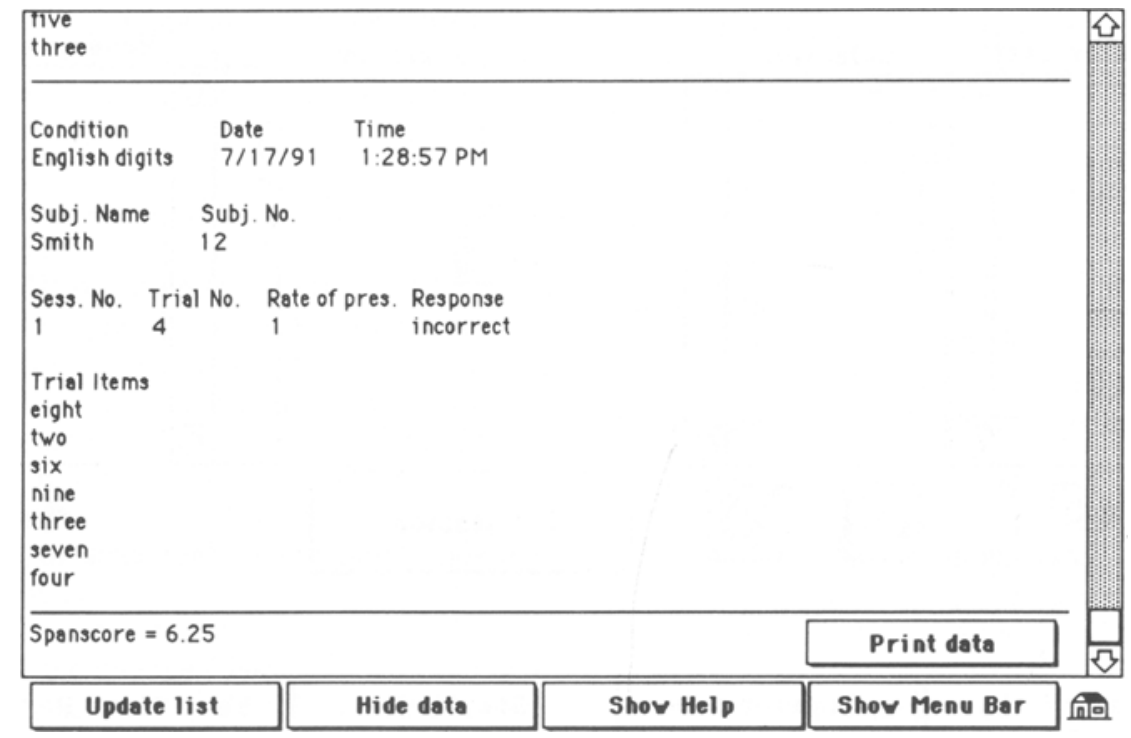

Figure 2. STM Experimenter: sample data file. 
$(\$ 10.00)$ is requested to help defray costs. For $£ 10$ (US\$20), a second disk of high fidelity prerecorded stimuli will be included (one-, two-, and three-syllable words/ non-words, digits in English and French).

\section{REFERENCES}

Baddeley, A., Thomson, N., \& Buchanan, M. (1975). Word length and the structure of short term memory. Journal of Verbal Learning \& Verbal Behavior, 14, 575-589.

ChUTE, D. (1989). MacLaboratory Psychology Version 1.0 [computer program]. Devon, PA: MacLaboratory. (314 Exeter Rd., Devon, PA 19333).

ConRaD, R. (1963). Acoustic confusions and memory span for words. Nature, 197, 1029-1030.

Costin, D. (1988). MacLab: A Macintosh system for psychology labs. Behavior Research Methods, Instruments, \& Computers, 20, 197-200.

Dougherty, T. J. (1990). Contour: A hypermedia environment for teaching about subjective contours and other visual illusions. Behavior Research Methods, Instruments, \& Computers, 22, 223-227.

Ebbinghaus, H. (1885). Memory: A contribution to experimental psychology. New York: Columbia University Press.

Geisler-Brenstein, E., \& Brenstein, R. J. (1989). The potential of HyperCard for psychological research and instruction: A general dis- cussion and description of two research applications. Behavior Research Methods, Instruments, \& Computers, 21, 307-311.

Gibson, J. M. (1987). Using digitized auditory stimuli on the Macintosh computer. Behavior Research Methods, Instruments, \& Computers, 19, 257-259.

Gum, T., Bun, D. (1988). PsychLab Manual. Montreal, Quebec: Montreal Neurological Institute.

JACOBS, J. (1887). Experiments on prehension. Mind, 12, 75-79

MacRecorder [Sound digitizer] (1990). Emeryville, CA: Farallon Computing.

Martin, T. A., \& Allan, W. E. (1991). An evaluation of touch-screen input for a HyperCard-based digit-span task. Behavior Research Methods, Instruments, \& Computers, 23, 253-255.

Nason, S., Zabrucky, K. (1988). A program for comprehension monitoring of text using HyperCard for the Macintosh. Behavior Research Methods, Instruments, \& Computers, 20, 499-502.

REEKES, J., \& ThOMPSON, T. (1991, July). Macintosh sound revealed. Byte, pp. 249-262.

TVERSKY, B. (1990). Experiments in cognitive psychology [computer program]. Stanford, CA: Stanford University Software Distribution Center.

(Manuscript received April 29, 1991; revision accepted for publication June 10, 1992.) 